\title{
On Generating All Minimal Integer Solutions for a Monotone System of Linear Inequalities ${ }^{\star}$
}

\author{
E. Boros ${ }^{1}$, K. Elbassioni ${ }^{2}$, V. Gurvich ${ }^{1}$, L. Khachiyan ${ }^{2}$, and K. Makino ${ }^{3}$
}

1 RUTCOR, Rutgers University, 640 Bartholomew Road, Piscataway NJ 08854-8003;

\{boros, gurvich\}@rutcor.rutgers.edu

2 Department of Computer Science, Rutgers University, 110 Frelinghuysen Road,

Piscataway NJ 08854-8003; elbassio@paul.rutgers.edu, leonid@cs.rutgers.edu

3 Division of Systems Science, Graduate School of Engineering Science, Osaka

University, Toyonaka, Osaka, 560-8531, Japan; makino@sys.es.osaka-u.ac.jp

\begin{abstract}
We consider the problem of enumerating all minimal integer solutions of a monotone system of linear inequalities. We first show that for any monotone system of $r$ linear inequalities in $n$ variables, the number of maximal infeasible integer vectors is at most $r n$ times the number of minimal integer solutions to the system. This bound is accurate up to a polylog $(r)$ factor and leads to a polynomial-time reduction of the enumeration problem to a natural generalization of the well-known dualization problem for hypergraphs, in which dual pairs of hypergraphs are replaced by dual collections of integer vectors in a box. We provide a quasi-polynomial algorithm for the latter dualization problem. These results imply, in particular, that the problem of incrementally generating minimal integer solutions of a monotone system of linear inequalities can be done in quasi-polynomial time.
\end{abstract}

Keywords: Integer programming, complexity of incremental algorithms, dualization, quasi-polynomial time, monotone discrete binary functions, monotone inequalities, regular discrete functions.

\section{Introduction}

Consider a system of $r$ linear inequalities in $n$ integer variables

$$
A x \geq b, \quad x \in \mathcal{C}=\left\{x \in \mathbb{Z}^{n} \mid 0 \leq x \leq c\right\}
$$

where $A$ is a rational $r \times n$-matrix, $b$ is a rational $r$-vector, and $c$ is a non-negative integral $n$-vector some or all of whose components may be infinite. We assume that (1) is a monotone system of inequalities: if $x \in \mathcal{C}$ satisfies (1) then any vector $y \in \mathcal{C}$ such that $y \geq x$ is also feasible. For instance, (11) is monotone if the

\footnotetext{
* The research of the first and third authors was supported in part by the Office of Naval Research (Grant N00014-92-J-1375), and the National Science Foundation (Grant DMS 98-06389). The research of the third and forth authors was supported in part by the National Science Foundation (Grant CCR-9618796).
}

F. Orejas, P.G. Spirakis, and J. van Leeuwen (Eds.): ICALP 2001, LNCS 2076, pp. 92-103 2001.

(C) Springer-Verlag Berlin Heidelberg 2001 
matrix $A$ is non-negative. Let us denote by $\mathcal{F}=\mathcal{F}_{A, b, c}$ the set of all minimal feasible integral vectors for (1), i.e. $y \in \mathcal{F}$ if there is no solution $x$ of (1) such that $x \leq y, x \neq y$. In particular, we have $\{x \in \mathcal{C} \mid A x \geq b\}=\bigcup_{y \in \mathcal{F}}\{x \in \mathcal{C} \mid x \geq y\}$. In this paper, we are concerned with the problem of incrementally generating $\mathcal{F}$ :

$G E N\left(\mathcal{F}_{A, b, c}, \mathcal{X}\right):$ Given a monotone system [1) and a set $\mathcal{X} \subseteq \mathcal{F}_{A, b, c}$ of minimal feasible vectors for (1), either find a new minimal integral vector satisfying (1), or show that $\mathcal{X}=\mathcal{F}_{A, b, c}$.

The entire set $\mathcal{F}=\mathcal{F}_{A, b, c}$ can be constructed by initializing $\mathcal{X}=\emptyset$ and iteratively solving the above problem $|\mathcal{F}|+1$ times.

If $A$ is a binary matrix, and $b, c$ are vectors of all ones, then $\mathcal{F}$ is the set of (characteristic vectors of) all minimal transversals to the hypergraph defined by the rows of $A$. In this case, problem $G E N\left(\mathcal{F}_{A, b, c}, \mathcal{X}\right)$ turns into the wellknown hypergraph dualization problem: incrementally enumerate all the minimal transversals (equivalently, all the maximal independent sets) for a given hypergraph (see, e.g., [3,10]). Some applications of the hypergraph dualization problem are discussed in 179. The case where $A$ is binary, $c$ is the vector of all ones and $b$ is arbitrary, is equivalent with the generation of so-called multiple transversals [5]. If $A$ is integral and $c=+\infty$, the generation of $\mathcal{F}$ can also be regarded as the computation of the Hilbert basis for the ideal $\left\{x \in \mathbb{Z}^{n} \mid A x \geq b, x \geq 0\right\}$. One more application of problem $G E N\left(\mathcal{F}_{A, b, c}, \mathcal{X}\right)$ is related to stochastic programming, more precisely to the generation of minimal $p$-efficient points for a given probability distribution of a discrete random variable $\xi \in \mathbb{Z}^{n}$. An integer vector $y \in \mathbb{Z}^{n}$ is called $p$-efficient, if $\operatorname{Prob}(\xi \leq y) \geq p$. It is known that for every probability distribution and every $p>0$ there are finitely many minimal $p$-efficient points, furthermore that for $r$-concave probability distributions these points are exactly the minimal integral points of a corresponding convex monotone system (see, e.g., [14]).

Let $J^{*}=\left\{j \mid c_{j}=\infty\right\}$ and $J_{*}=\{1, \ldots, n\} \backslash J^{*}$ be, respectively, the sets of unbounded and bounded integer variables in (11). Consider an arbitrary vector $x=\left(x_{1}, \ldots, x_{n}\right) \in \mathcal{F}_{A, b, c}$ such that $x_{j}>0$ for some $j \in J^{*}$. Then it is easy to see that

$$
x_{j} \leq \max _{i: a_{i j}>0}\left\lceil\frac{b_{i}-\sum_{k \in J_{*}} \min \left\{0, a_{i k}\right\} c_{k}}{a_{i j}}\right\rceil<+\infty .
$$

Since the bounds of (2) are easy to compute, and since appending these bounds to (II) does not change the set $\mathcal{F}_{A, b, c}$, we shall assume in the sequel that all components of the non-negative vector $c$ are finite, even though this may not be the case for the original system. This assumption does not entail any loss of generality and allows us to consider $\mathcal{F}_{A, b, c}$ as a system of integral vectors in a finite box. We shall also assume that the input monotone system (11) is feasible, i.e., $\mathcal{F}_{A, b, c} \neq \emptyset$. For a finite and non-negative $c$ this is equivalent to $A c \geq b$. 
Let $\mathcal{A}$ be a collection of integral vectors in $\mathcal{C}$ and let $\mathcal{A}^{+}=\{x \in \mathcal{C} \mid x \geq$ $a$ for some $a \in \mathcal{A}\}$ and $\mathcal{A}^{-}=\{x \in \mathcal{C} \mid x \leq a$ for some $a \in \mathcal{A}\}$ denote the ideal and filter generated by $\mathcal{A}$. Any element in $\mathcal{C} \backslash \mathcal{A}^{+}$is called independent of $\mathcal{A}$. Let $\mathcal{I}(\mathcal{A})$ be the set of all maximal independent elements for $\mathcal{A}$, then for any finite box $\mathcal{C}$ we have the decomposition:

$$
\mathcal{A}^{+} \cap \mathcal{I}(\mathcal{A})^{-}=\emptyset, \quad \mathcal{A}^{+} \cup \mathcal{I}(\mathcal{A})^{-}=\mathcal{C}
$$

In particular, if $\mathcal{A}$ is the set $\mathcal{F}=\mathcal{F}_{A, b, c}$ of all minimal feasible integral vectors for (1), then the ideal $\mathcal{F}^{+}$is the solution set of (1), while the filter $\mathcal{C} \backslash \mathcal{F}^{+}$is generated by the set $\mathcal{I}(\mathcal{F})$ of all maximal infeasible integral vectors for (1):

$$
\{x \in \mathcal{C} \mid A x \nsupseteq b\}=\bigcup_{y \in \mathcal{I}(\mathcal{F})}\{y\}^{-} .
$$

It is known that the problem of incrementally generating all maximal infeasible vectors for (1) is NP-hard even if $c$ is the vector of all ones and the matrix $A$ is binary:

Proposition 1 (c.f. [12]). Given a binary matrix $A$ and a set $\mathcal{X} \subseteq \mathcal{I}\left(\mathcal{F}_{A, b, c}\right)$ of maximal infeasible Boolean vectors for $A x \geq b, x \in\{0,1\}^{n}$, it is NP-complete to decide if the set $\mathcal{X}$ can be extended, that is if $\mathcal{I}\left(\mathcal{F}_{A, b, c}\right) \backslash \mathcal{X} \neq \emptyset$.

In contrast to that, we show in this paper that the problem of incrementally generating all minimal feasible vectors for (1) is unlikely to be NP-hard.

Theorem 1. Problem $G E N\left(\mathcal{F}_{A, b, c}, \mathcal{X}\right)$ can be solved in quasi-polynomial time poly $(\mid$ input $\mid)+t^{o(\log t)}$, where $t=\max \{n, r,|\mathcal{X}|\}$.

It was conjectured in [1] that problem $\operatorname{GEN}\left(\mathcal{F}_{A, b, c}, \mathcal{X}\right)$ cannot be solved in polynomial time unless $\mathrm{P}=\mathrm{NP}$.

To prove Theorem 1 we first bound the number of maximal infeasible vectors for (11) in terms of the dimension of the system and the number of minimal feasible vectors.

Theorem 2. Suppose that the monotone system (1) is feasible, i.e., $A c \geq b$. Then for any non-empty set $\mathcal{X} \subseteq \mathcal{F}_{A, b, c}$ we have

$$
\left|\mathcal{I}(\mathcal{X}) \cap \mathcal{I}\left(\mathcal{F}_{A, b, c}\right)\right| \leq r \sum_{x \in \mathcal{X}} p(x),
$$

where $p(x)$ is the number of positive components of $x$. In particular,

$$
\left|\mathcal{I}(\mathcal{X}) \cap \mathcal{I}\left(\mathcal{F}_{A, b, c}\right)\right| \leq r n|\mathcal{X}|
$$

which for $\mathcal{X}=\mathcal{F}_{A, b, c}$ leads to the inequality $\left|\mathcal{I}\left(\mathcal{F}_{A, b, c}\right)\right| \leq r n\left|\mathcal{F}_{A, b, c}\right|$. 
It should be mentioned that the bounds of Theorem 2 are sharp for $r=1$, e.g., for the inequality $x_{1}+\ldots+x_{n} \geq n$. For large $r$, these bounds are accurate up to a factor poly-logarithmic in $r$. To see this, let $n=2 k$ and consider the monotone system of $r=2^{k}$ inequalities of the form

$$
x_{i_{1}}+x_{i_{2}}+\cdots+x_{i_{k}} \geq 1, i_{1} \in\{1,2\}, i_{2} \in\{3,4\}, \ldots, i_{k} \in\{2 k-1,2 k\},
$$

where $x=\left(x_{1}, \ldots, x_{n}\right) \in \mathcal{C}=\left\{x \in \mathbb{Z}^{n} \mid 0 \leq x \leq c\right\}$. For any positive integral vector $c$, this system has $2^{k}$ maximal infeasible integral vectors and only $k$ minimal feasible integral vectors, i.e.,

$$
\left|\mathcal{I}\left(\mathcal{F}_{A, b, c}\right)\right|=\frac{r n}{2(\log r)^{2}}\left|\mathcal{F}_{A, b, c}\right| .
$$

Needless to say that in general, $\left|\mathcal{F}_{A, b, c}\right|$ cannot be bounded by a polynomial in $r, n$, and $\left|\mathcal{I}\left(\mathcal{F}_{A, b, c}\right)\right|$. For instance, for $n=2 k$ the system of $k$ inequalities $x_{1}+x_{2} \geq 1, \quad x_{3}+x_{4} \geq 1, \ldots, x_{2 k-1}+x_{2 k} \geq 1$ has $2^{k}$ minimal feasible binary vectors and only $k$ maximal infeasible binary vectors.

Let us add finally that if the number of inequalities in (1) is fixed, then $\left|\mathcal{F}_{A, b, c}\right|$ can also be polynomially bounded by $\left|\mathcal{I}\left(\mathcal{F}_{A, b, c}\right)\right|$, and accordingly, the set of all maximal infeasible integer vectors for (1) can be generated in quasipolynomial time. In other words, Proposition 1 cannot hold for $r=$ const unless any problem in NP can be solved in quasi-polynomial time. Furthermore, for systems with fixed number of non-zero coefficients per inequality and bounded box size, problem $G E N\left(\mathcal{F}_{A, b, c}, \mathcal{X}\right)$ can be efficiently solved in parallel (see [4]).

We prove Theorem 2 in Section 2, and then use this theorem in the next section to reduce problem $G E N\left(\mathcal{F}_{A, b, c}, \mathcal{X}\right)$ to a natural generalization of the hypergraph dualization problem. Our generalized dualization problem replaces hypergraphs by collections of integer vectors in a box.

Theorem 3. $G E N\left(\mathcal{F}_{A, b, c}, \mathcal{X}\right)$ is polynomial-time reducible to the following problem:

$\operatorname{DU} A L(\mathcal{C}, \mathcal{A}, \mathcal{B})$ : Given an integral box $\mathcal{C}$, a family of vectors $\mathcal{A} \subseteq \mathcal{C}$, and a collection of maximal independent elements $\mathcal{B} \subseteq \mathcal{I}(\mathcal{A})$, either find a new maximal independent element $x \in \mathcal{I}(\mathcal{A}) \backslash \mathcal{B}$, or prove that $\mathcal{B}=\mathcal{I}(\mathcal{A})$.

Note that for $\mathcal{C}=\{0,1\}^{n}$, problem $\operatorname{DU} A L(\mathcal{C}, \mathcal{A}, \mathcal{B})$ turns into the hypergraph dualization problem. Other applications of the dualization problem on boxes can be found in 26,13. In Section 4 we extend the hypergraph dualization algorithm of [8] to problem $D U A L(\mathcal{C}, \mathcal{A}, \mathcal{B})$ and show that the latter problem can be solved in quasi-polynomial time:

Theorem 4. Given two sets $\mathcal{A}$, and $\mathcal{B} \subseteq \mathcal{I}(\mathcal{A})$ in an integral box $\mathcal{C}=\{x \in$ $\left.\mathbb{Z}^{n} \mid 0 \leq x \leq c\right\}$, problem $D U A L(\mathcal{C}, \mathcal{A}, \mathcal{B})$ can be solved in poly $(n, m)+m^{o(\log m)}$ time, where $m=|\mathcal{A}|+|\mathcal{B}|$.

Clearly, Theorem 1 follows from Theorems 3 and 4 . The special cases of Theorems 2 and 3 for Boolean systems $x \in\{0,1\}^{n}$ can be found in [5].

The remainder of the paper consists of the proofs of Theorems 2 [3and 4 in Sections 2 [ 4 respectively. 


\section{Bounding the Number of Maximal Infeasible Vectors}

In this section we prove Theorem 2 We first need some notations and definitions.

Let $\mathcal{C}=\left\{x \in \mathbb{Z}^{n} \mid 0 \leq x \leq c\right\}$ be a box and let $f: \mathcal{C} \rightarrow\{0,1\}$ be a discrete binary function. The function $f$ is called monotone if $f(x) \geq f(y)$ whenever $x \geq y$ and $x, y \in \mathcal{C}$. We denote by $T(f)$ and $F(f)$ the sets of all true and all false vectors of $f$, i.e.,

$$
T(f)=\{x \in \mathcal{C} \mid f(x)=1\}=(\min [f])^{+}, \quad F(f)=\{x \in \mathcal{C} \mid f(x)=0\}=(\max [f])^{-},
$$

where $\min [f]$ and $\max [f]$ are the sets of all minimal true and all maximal false vectors of $f$, respectively.

Let $\sigma \in \mathbb{S}_{n}$ be a permutation of the coordinates and let $x, y$ be two $n$-vectors. We say that $y$ is a left-shift of $x$ and write $y \succeq_{\sigma} x$ if the inequalities

$$
\sum_{j=1}^{k} y_{\sigma_{j}} \geq \sum_{j=1}^{k} x_{\sigma_{j}}
$$

hold for all $k=1, \ldots, n$. A discrete binary function $f: \mathcal{C} \rightarrow\{0,1\}$ is called 2-monotonic with respect to $\sigma$ if $f(y) \geq f(x)$ whenever $y \succeq_{\sigma} x$ and $x, y \in \mathcal{C}$. Clearly, $y \geq x$ implies $y \succeq_{\sigma} x$ for any $\sigma \in \mathbb{S}_{n}$, so that any 2-monotonic function is monotone.

The function $f$ will be called regular if it is 2-monotonic with respect to the identity permutation $\sigma=(1,2, \ldots, n)$. Any 2-monotonic function can be transformed into a regular one by appropriately re-indexing its variables. To simplify notations, we shall state Lemma 1 below for regular functions, i.e., we fix $\sigma=(1,2, \ldots, n)$ in this lemma.

For a given subset $\mathcal{A} \subseteq \mathcal{C}$ let us denote by $\mathcal{A}^{*}$ all the vectors which are leftshifts of some vectors of $\mathcal{A}$, i.e., $\mathcal{A}^{*}=\{y \in \mathcal{C} \mid y \succeq x$ for some $x \in \mathcal{A}\}$. Clearly, $T(f)=(\min [f])^{*}$ for a regular function $f$ (in fact, the subfamily of right-most vectors of $\min [f]$ would be enough to use here.)

Given monotone discrete functions $f$ and $g$, we call $g$ a regular majorant of $f$, if $g(x) \geq f(x)$ for all $x \in \mathcal{C}$, and $g$ is regular. Clearly, $T(g) \supseteq(\min [f])^{*}$ must hold in this case, and the discrete function $h$ defined by $T(h)=(\min [f])^{*}$ is the unique minimal regular majorant of $f$.

For a vector $x \in \mathcal{C}$, and for an index $1 \leq k \leq n$, let the vectors $x^{(k]}$ and $x^{[k)}$ be defined by

$$
x_{j}^{(k]}= \begin{cases}x_{j} & \text { for } j \leq k, \\ 0 & \text { otherwise }\end{cases}
$$

and

$$
x_{j}^{[k)}= \begin{cases}x_{j} & \text { for } j \geq k, \\ 0 & \text { otherwise. }\end{cases}
$$

Let us denote by $e$ the $n$-vector of all 1's, let $e_{j}$ denote the $j^{\text {th }}$ unit vector, $j=1, \ldots, n$, and let $p(x)$ denote the number of positive components of the vector $x \in \mathcal{C}$. 
Lemma 1. Given a monotone discrete binary function $f: \mathcal{C} \rightarrow\{0,1\}$ such that $f \not \equiv 0$, and a regular majorant $g \geq f$, we have the inequality

$$
|F(g) \cap \max [f]| \leq \sum_{x \in \min [f]} p(x) .
$$

Proof. Let us denote by $h$ the unique minimal regular majorant of $f$. Then we have $F(g) \cap \max [f] \subseteq F(h) \cap \max [f]$, and hence it is enough to show the statement for $g=h$, i.e. when $T(g)=(\min [f])^{*}$.

For a vector $y \in \mathcal{C} \backslash\{c\}$ let us denote by $l=l_{y}$ the index of the last component which is less than $c_{l}$, i.e., $l=\max \left\{j \mid y_{j}<c_{j}\right\} \in\{1, \ldots, n\}$. We claim that for every $y \in F(h) \cap \max [f]$ there exists an $x \in \min [f]$ such that

$$
y=x^{(l-1]}+\left(x_{l}-1\right) e_{l}+c^{[l+1)},
$$

where $l=l_{y}$. To see this claim, first observe that $y \neq c$ because $y \in F(f)$ and $f \not \equiv 0$. Second, for any $j$ with $y_{j}<c_{j}$ we know that $y+e_{j} \in T(f)$, by the definition of a maximal false point. Hence there exists a minimal true-vector $x \in \min [f]$ such that $x \leq y+e_{l}$ for $l=l_{y}$. We must have $x^{(l-1]}=y^{(l-1]}$, since if $x_{i}<y_{i}$ for some $i<l$, then $y \geq x+e_{i}-e_{l} \succeq x$ would hold, i.e. $y \succeq x$ would follow, implying $y \in(\min [f])^{*}$ and yielding a contradiction with $y \in F(h)=$ $\mathcal{C} \backslash(\min [f])^{*}$. Finally, the definition of $l=l_{y}$ implies that $y^{[l+1)}=c^{[l+1)}$. Hence, our claim and the equality (6) follow.

The above claim implies that

$$
F(h) \cap \max [f] \subseteq\left\{x^{(l-1]}+\left(x_{l}-1\right) e_{l}+c^{[l+1)} \mid x \in \min [f], x_{l}>0\right\},
$$

and hence (5) and thus the lemma follow.

Lemma 2. Let $f: \mathcal{C} \rightarrow\{0,1\}$ be a monotone discrete binary function such that $f \not \equiv 0$ and

$$
x \in T(f) \Rightarrow \alpha x \stackrel{\text { def }}{=} \alpha_{1} x_{1}+\ldots \alpha_{n} x_{n} \geq \beta
$$

where $\alpha=\left(\alpha_{1}, \ldots, \alpha_{n}\right)$ is a given real vector and $\beta$ is a real threshold. Then

$$
|\{x \in \mathcal{C} \mid \alpha x<\beta\} \cap \max [f]| \leq \sum_{x \in \min [f]} p(x)
$$

Proof. Suppose that some of the weights $\alpha_{1}, \ldots, \alpha_{n}$ are negative, say $\alpha_{1}<$ $0, \ldots, \alpha_{k}<0$ and $\alpha^{[k+1)} \geq 0$. Since $\alpha x \geq \beta$ for any $x \in T(f)$ and since $f$ is monotone, we have $x \in T(f) \Rightarrow \alpha^{[k+1)} x \geq \beta-\alpha^{(k]} c^{(k]}$. For any $x \in \mathcal{C}$ we also have $\{x \mid \alpha x<\beta\} \subseteq\left\{x \mid \alpha^{[k+1)} x<\beta-\alpha^{(k]} c^{(k]}\right\}$. Hence it suffices to prove the lemma for the non-negative weight vector $\alpha^{[k+1)}$ and the threshold $\beta-\alpha^{(k]} c^{(k]}$. In other words, we can assume without loss of generality that the original weight vector $\alpha$ is non-negative. 
Let $\sigma \in \mathbb{S}^{n}$ be a permutation such that $\alpha_{\sigma_{1}} \geq \alpha_{\sigma_{2}} \geq \cdots \geq \alpha_{\sigma_{n}} \geq 0$. Then the threshold function

$$
g(x)= \begin{cases}1 & \text { if } \alpha x \geq \beta \\ 0 & \text { otherwise }\end{cases}
$$

is 2 -monotonic with respect to $\sigma$. By (7), we have $g \geq f$ for all $x \in \mathcal{C}$, i.e., $g$ majorates $f$. In addition, $F(g)=\{x \in \mathcal{C} \mid \alpha x<\beta\}$, and hence Lemma 2 follows from Lemma 1.

We are now ready to show inequality (41) and finish the proof of Theorem 2. Given a non-empty set $\mathcal{X} \subseteq \mathcal{F}_{A, b, c}$, consider the monotone discrete function $f: \mathcal{C} \rightarrow\{0,1\}$ defined by the condition $\min [f]=\mathcal{X}$. Since (11) is monotone, any true vector of $f$ also satisfies (11):

$$
x \in T(f) \Rightarrow a_{k 1} x_{1}+\ldots+a_{k n} x_{n} \geq b_{k}
$$

for all $k=1, \ldots, r$. In addition, $f \neq \equiv 0$ because $\mathcal{X} \neq \emptyset$. Thus, by Lemma 2 we have the inequalities

$$
\left|\left\{x \mid a_{k 1} x_{1}+\ldots+a_{k n} x_{n}<b_{k}\right\} \cap \max [f]\right| \leq \sum_{x \in \mathcal{X}} p(x)
$$

for each $k=1, \ldots, r$. Now, from $\max [f]=\mathcal{I}(\mathcal{X})$ we deduce that

$$
\mathcal{I}\left(\mathcal{F}_{A, b, c}\right) \cap \mathcal{I}(\mathcal{X}) \subseteq \bigcup_{k=1}^{r}\left\{x \mid a_{k 1} x_{1}+\ldots+a_{k n} x_{n}<b_{k}\right\} \cap \max [f]
$$

and thus (4) and the theorem follow by (81).

\section{Generating Minimal Integer Solutions via Integral Dualization}

The proof of Theorem 3 has two ingredients. First, we show that given a monotone system (11), the sets $\mathcal{I}\left(\mathcal{F}_{A, b, c}\right)$ and $\mathcal{F}_{A, b, c}$ can be jointly enumerated by iteratively solving the dualization problem $\operatorname{DU} A L(\mathcal{C}, \mathcal{A}, \mathcal{B})$ introduced in Theorem 3. Second, we invoke Theorem 2 and argue that since the number of maximal infeasible vectors is relatively small, the generation of $\mathcal{F}_{A, b, c}$ polynomially reduces to the joint generation of $\mathcal{I}\left(\mathcal{F}_{A, b, c}\right)$ and $\mathcal{F}_{A, b, c}$.

\subsection{Joint Generation of Dual Subsets in an Integral Box}

Let $\mathcal{F}=\mathcal{F}_{A, b, c}$ be the set of minimal integral vectors for (11), and consider the following problem of jointly generating all points of $\mathcal{F}$ and $\mathcal{I}(\mathcal{F})$ :

$G E N(\mathcal{F}, \mathcal{I}(\mathcal{F}), \mathcal{A}, \mathcal{B}):$ Given two explicitly listed collections $\mathcal{A} \subseteq \mathcal{F}$ and $\mathcal{B} \subseteq$ $\mathcal{I}(\mathcal{F})$, either find a new point in $(\mathcal{F} \backslash \mathcal{A}) \cup(\mathcal{I}(\mathcal{F}) \backslash \mathcal{B})$, or prove that these collections are complete: $(\mathcal{A}, \mathcal{B})=(\mathcal{F}, \mathcal{I}(\mathcal{F}))$. 
Proposition 2. Problem $G E N(\mathcal{F}, \mathcal{I}(\mathcal{F}), \mathcal{A}, \mathcal{B})$ can be solved in time poly $(n$, $\left.|\mathcal{A}|,|\mathcal{B}|, \log \|c\|_{\infty}\right)+T_{\text {dual }}$, where $T_{\text {dual }}$ denotes the time required to solve problem $\operatorname{DUAL}(\mathcal{C}, \mathcal{A}, \mathcal{B})$.

Proof. The reduction is via the following Algorithm $\mathcal{J}$ :

Step 1. Check whether $\mathcal{B} \subseteq \mathcal{I}(\mathcal{A})$. If there is an $x \in \mathcal{B} \backslash \mathcal{I}(\mathcal{A})$, then $x \notin \mathcal{F}^{+}$ because $x \in \mathcal{B} \subseteq \mathcal{I}(\mathcal{F})$. This and the inclusion $\mathcal{A} \subseteq \mathcal{F}$ imply that $x \notin \mathcal{A}^{+}$. Since $x \notin \mathcal{I}(\mathcal{A})$, we can find a coordinate $j \in\{1, \ldots, n\}$ for which $y=x+e_{j} \notin \mathcal{A}^{+}$. By the maximality of $x$ in $\mathcal{C} \backslash \mathcal{F}^{+}, y$ belongs to $\mathcal{F}^{+}$and therefore, there must exist a $z \in \mathcal{F}$ such that $z \leq y$. Since $z \notin \mathcal{A}^{+}$, we have $z \in \mathcal{F} \backslash \mathcal{A}$, i.e., $z$ is a new minimal integral vector in $\mathcal{F}$ which can be found in $\operatorname{poly}\left(n,|\mathcal{A}|,|\mathcal{B}|, \log \|c\|_{\infty}\right)$ time by performing coordinate binary searches on the box $\left\{z \in \mathbb{Z}^{n} \mid 0 \leq z \leq y\right\}$.

Step 2 is similar to the previous step: we check whether $\mathcal{A} \subseteq \mathcal{I}^{-1}(\mathcal{B})$, where $\mathcal{I}^{-1}(\mathcal{B})$ is the set of integral vectors minimal in $\mathcal{C} \backslash \mathcal{B}^{-}$. If $\mathcal{A}$ contains an element that is not minimal in $\mathcal{C} \backslash \mathcal{B}^{-}$, we can find a new point in $\mathcal{I}(\mathcal{F}) \backslash \mathcal{B}$ and halt.

Step 3. Suppose that $\mathcal{B} \subseteq \mathcal{I}(\mathcal{A})$ and $\mathcal{A} \subseteq \mathcal{I}^{-1}(\mathcal{B})$. Then $(\mathcal{A}, \mathcal{B})=(\mathcal{F}, \mathcal{I}(\mathcal{F})) \Leftrightarrow$ $\mathcal{B}=\mathcal{I}(\mathcal{A})$. (To see this, assume that $\mathcal{B}=\mathcal{I}(\mathcal{A})$, and suppose on the contrary that there is an $x \in \mathcal{F} \backslash \mathcal{A}$. Since $x \notin \mathcal{A}=\mathcal{I}^{-1}(\mathcal{B})$ and $x \notin \mathcal{B}^{-} \subseteq \mathcal{I}(\mathcal{F})^{-}$, there must exist a $y \in \mathcal{I}^{-1}(\mathcal{B})=\mathcal{A} \subseteq \mathcal{F}$ such that $y \leq x$. Hence we get two distinct elements $x, y \in \mathcal{F}$ such that $y \leq x$, which contradicts the definition of $\mathcal{F}$. The existence of an $x \in \mathcal{I}(\mathcal{F}) \backslash \mathcal{B}$ leads to a similar contradiction.) To check the condition $\mathcal{B}=\mathcal{I}(\mathcal{A})$, we solve problem $D U A L(\mathcal{C}, \mathcal{A}, \mathcal{B})$. If $\mathcal{B} \neq \mathcal{I}(\mathcal{A})$, we obtain a new point $x \in \mathcal{I}(\mathcal{A}) \backslash \mathcal{B}$. By (3), either $x \in \mathcal{F}^{+}$, or $x \in \mathcal{I}(\mathcal{F})^{-}$and we can decide which of these two cases holds by checking the feasibility of $x$ for (11). In the first case, we obtain a new point $y \in\{x\}^{-} \cap(\mathcal{F} \backslash \mathcal{A})$ by performing binary searches on the coordinates of the box $\left\{y \in \mathbb{Z}^{n} \mid 0 \leq y \leq x\right\}$. In the second case, a new point in $\{x\}^{+} \cap(\mathcal{I}(\mathcal{F}) \backslash \mathcal{B})$ can be obtained by searching the box $\left\{y \in \mathbb{Z}^{n} \mid x \leq y \leq c\right\}$.

Let $\mathcal{F} \subseteq \mathcal{C}$ be an arbitrary antichain, i.e., a system of integral vectors such that $x \not \leq y$ for any two distinct elements $x, y \in \mathcal{F}$. It is easy to see that Algorithm $\mathcal{J}$ and Proposition 2 can be used for any class of antichains $\mathcal{F}$ defined by a polynomial-time membership oracle for $\mathcal{F}^{+}$.

\subsection{Uniformly Dual-Bounded Antichains}

Extending the definition of dual-bounded hypergraphs in [5], we say that (a class of antichains) $\mathcal{F} \subseteq \mathcal{C}$ is uniformly dual-bounded if there exists a polynomial $p$ such that, for any nonempty subset $\mathcal{X} \subseteq \mathcal{F}$, we have

$$
|\mathcal{I}(\mathcal{F}) \cap \mathcal{I}(\mathcal{X})| \leq p(|\mathcal{X}|)
$$

Proposition 3. Suppose that $\mathcal{F}$ is uniformly dual-bounded and there exists a polynomial-time membership oracle for $\mathcal{F}^{+}$. Then problem $\operatorname{GEN}(\mathcal{F})$ is polynomial-time reducible to problem $\operatorname{DU} A L(\mathcal{C}, \mathcal{A}, \mathcal{B})$. 
Proof. Given a set $\mathcal{X}$ in $\mathcal{F}$, we repeatedly run Algorithm $\mathcal{J}$ until it either produces a new element in $\mathcal{F} \backslash \mathcal{X}$ or proves that $\mathcal{X}=\mathcal{F}$ by generating the entire family $\mathcal{I}(\mathcal{F})$. By Step 1, as long as Algorithm $\mathcal{J}$ outputs elements of $\mathcal{I}(\mathcal{F})$, these elements also belong to $\mathcal{I}(\mathcal{X})$, and hence the total number of such elements does not exceed $p(|\mathcal{X}|)$.

By Theorem 2, the set of minimal integral solutions to any monotone system of linear inequalities is uniformly-dual bounded, and hence Theorem 3 is a corollary of Proposition 3 .

\section{Dualization in Products of Chains}

Let $\mathcal{C} \stackrel{\text { def }}{=} \mathcal{C}_{1} \times \ldots \times \mathcal{C}_{n}$ be an integer box defined by the product of $n$ chains $\mathcal{C}_{i}=\left[l_{i}: u_{i}\right]$ where $l_{i}, u_{i} \in \mathbb{Z}$ are, respectively, the lower and upper bounds of chain $\mathcal{C}_{i}$. Given an antichain $\mathcal{A} \subseteq \mathcal{C}$, and an antichain $\mathcal{B} \subseteq \mathcal{I}(\mathcal{A})$, we say that $\mathcal{B}$ is dual to $\mathcal{A}$ if $\mathcal{B}=\mathcal{I}(\mathcal{A})$, i.e., $\mathcal{B}$ contains all the maximal elements of $\mathcal{C} \backslash \mathcal{A}^{+}$. If $\mathcal{C}$ is the unit cube, we obtain the familiar notion of dual hypergraphs, where $\mathcal{I}(\mathcal{A})$ becomes the complementary set of the transversal hypergraph of $\mathcal{A}$. In this section, we show how to extend the hypergraph dualization algorithm of 8 ] to arbitrary systems $\mathcal{A}$ of integral vectors in a box $\mathcal{C}$.

As in [8], we shall analyze the running time of the algorithm in terms of the "volume" $v=v(\mathcal{A}, \mathcal{B}) \stackrel{\text { def }}{=}|\mathcal{A}||\mathcal{B}|$ of the input problem. In general, a given problem will be decomposed into a number of subproblems which we solve recursively. Since we have assumed that $\mathcal{B} \subseteq \mathcal{I}(\mathcal{A})$, (3) implies that the following condition holds for the original problem and all subsequent subproblems:

$$
a \not \leq b, \text { for all } a \in \mathcal{A}, b \in \mathcal{B} \text {. }
$$

Let $R(v)=R(v(\mathcal{A}, \mathcal{B}))$ denote the number of subproblems that have to be solved in order to solve the original problem, and let $m$ denote $|\mathcal{A}|+|\mathcal{B}|$, and $[n] \stackrel{\text { def }}{=}\{1, \ldots, n\}$. We start with the following proposition that provides the base case for recursion.

Proposition 4. Suppose $\min \{|\mathcal{A}|,|\mathcal{B}|\} \leq$ const, then problem $\operatorname{DU} A L(\mathcal{C}, \mathcal{A}, \mathcal{B})$ is solvable in polynomial time.

Proof. Let us assume without loss of generality that $\mathcal{B}=\left\{b^{1}, \ldots, b^{k}\right\}$, for some constant $k$. For $t \in[n]^{k}$ and $i \in[n]$, let $I_{i}^{t}=\left\{j \in[k] \mid t_{j}=i\right\}$. Then $\mathcal{C}=\mathcal{A}^{+} \cup \mathcal{B}^{-}$ if and only if for every $t \in[n]^{k}$ for which

$$
b_{i}^{j} \neq u_{i} \text {, for all } i \in[n], j \in I_{i}^{t},
$$

there exists an $a \in \mathcal{A}$ such that

$$
a_{i} \leq \max \left\{b_{i}^{j}+1 \mid j \in I_{i}^{t}\right\} \text { if } I_{i}^{t} \neq \emptyset, \text { and } a_{i}=l_{i} \text { otherwise. }
$$

To see this, assume first that $\mathcal{C}=\mathcal{A}^{+} \cup \mathcal{B}^{-}$and consider any $t \in[n]^{k}$ such that (10) holds. Let $x \in \mathcal{C}$ be defined by taking $x_{i}=\max \left\{b_{i}^{j}+1 \mid j \in I_{i}^{t}\right\}$ if $I_{i}^{t} \neq \emptyset$, 
and $x_{i}=l_{i}$ otherwise. Then $x \in \mathcal{C} \backslash \mathcal{B}^{-}$and hence $x \in \mathcal{A}^{+}$, implying that there is an $a \in \mathcal{A}$ satisfying (11). On the other hand, let us assume that for every $t \in[n]^{k}$ satisfying (10), there is an $a \in \mathcal{A}$ for which (11) holds. Consider an $x \in \mathcal{C} \backslash \mathcal{B}^{-}$, then there must exist, for every $j \in[k]$, a $t_{j} \in[n]$, such that $x_{t_{j}} \geq b_{t_{j}}^{j}+1$. Clearly $t=\left(t_{1}, \ldots, t_{k}\right) \in[n]^{k}$ satisfies (10), and therefore, there is an $a \in \mathcal{A}$ such that $a_{i} \leq \max \left\{b_{i}^{j}+1 \mid j \in I_{i}^{t}\right\} \leq x_{i}$ if $I_{i}^{t} \neq \emptyset$, and $a_{i}=l_{i}$ otherwise. This gives $x \in \mathcal{A}^{+}$.

Remark. Having found an $x \in \mathcal{C} \backslash\left(\mathcal{A}^{+} \cup \mathcal{B}^{-}\right)$, it is always possible to extend it to a maximal point with the same property in $O(n m \log m)$ time as follows. Let $\mathcal{Q}_{i}=\left\{a_{i}-1 \mid a \in \mathcal{A}\right\} \cup\left\{x_{i}, u_{i}\right\}, i=1, \ldots, n$, and assume that this list is kept in sorted order for each $i$. For $i=1, \ldots, n$, we iterate $x_{i} \leftarrow \max \{z \in$ $\left.\mathcal{Q}_{i} \mid\left(x_{1}, \ldots, x_{i-1}, z, x_{i+1}, \ldots, x_{n}\right) \notin \mathcal{A}^{+}\right\}$. Then the resulting point $x$ is maximal in $\mathcal{C} \backslash\left(\mathcal{A}^{+} \cup \mathcal{B}^{-}\right)$.

Now given two integral antichains $\mathcal{A}, \mathcal{B}$ that satisfy the necessary duality condition (9), we proceed as follows:

Step 1. If $\min \{|\mathcal{A}|,|\mathcal{B}|\} \leq 2$, the duality of $\mathcal{A}$ and $\mathcal{B}$ can be tested in $O\left(n^{3} m\right)$ time using Proposition 4 .

Step 2. For each $k \in[n]$ :

1. if $a_{k}>u_{k}$ for some $a \in \mathcal{A}\left(b_{k}<l_{k}\right.$ for some $\left.b \in \mathcal{B}\right)$, then $a$ (respectively, $b)$ can be clearly discarded from further consideration;

2. if $a_{k}<l_{k}$ for some $a \in \mathcal{A}\left(b_{k}>u_{k}\right.$ for some $\left.b \in \mathcal{B}\right)$, we set $a_{k} \leftarrow l_{k}$ (respectively, $b_{k} \leftarrow u_{k}$ ). Note that the duality condition (9) continues to hold after such replacements.

Thus we may assume for next steps that $\mathcal{A}, \mathcal{B} \subseteq \mathcal{C}$.

Step 3. Let $a^{o} \in \mathcal{A}, b^{o} \in \mathcal{B}$. By (9), there exists an $i \in[n]$, such that $a_{i}^{o}>b_{i}^{o}$. Assume, with no loss of generality, that $i=1$ and set $\mathcal{C}_{1}^{\prime} \leftarrow\left[a_{1}^{o}: u_{1}\right], \mathcal{C}_{1}^{\prime \prime} \leftarrow\left[l_{1}\right.$ : $\left.a_{1}^{o}-1\right]$. (Alternatively, we may set $\mathcal{C}_{1}^{\prime \prime} \leftarrow\left[l_{1}: b_{1}^{o}\right]$ and $\mathcal{C}_{1}^{\prime} \leftarrow\left[b_{1}^{o}+1: u_{1}\right]$.) Define

$$
\begin{aligned}
& \mathcal{A}^{\prime \prime}=\left\{a \in \mathcal{A} \mid a_{1}<a_{1}^{o}\right\}, \quad \mathcal{A}^{\prime}=\mathcal{A} \backslash \mathcal{A}^{\prime \prime}, \\
& \mathcal{B}^{\prime}=\left\{b \in \mathcal{B} \mid b_{1} \geq a_{1}^{o}\right\}, \quad \epsilon_{1}^{\mathcal{A}}=\frac{\left|\mathcal{A}^{\prime}\right|}{|\mathcal{A}|},
\end{aligned}
$$

Observe that $\epsilon_{1}^{\mathcal{A}}>0$ and $\epsilon_{1}^{\mathcal{B}}>0$ since $a^{o} \in \mathcal{A}^{\prime}$ and $b^{o} \in \mathcal{B}^{\prime \prime}$.

Denoting by $\mathcal{C}^{\prime}=\mathcal{C}_{1}^{\prime} \times \mathcal{C}_{2} \times \ldots \times \mathcal{C}_{n}$, and $\mathcal{C}^{\prime \prime}=\mathcal{C}_{1}^{\prime \prime} \times \mathcal{C}_{2} \times \ldots \times \mathcal{C}_{n}$ the two half-boxes of $\mathcal{C}$ induced by the above partitioning, it is then easy to see that $\mathcal{A}$ and $\mathcal{B}$ are dual in $\mathcal{C}$ if and only if

$$
\begin{aligned}
& \mathcal{A}, \mathcal{B}^{\prime} \text { are dual in } \mathcal{C}^{\prime}, \text { and } \\
& \mathcal{A}^{\prime \prime}, \mathcal{B} \text { are dual in } \mathcal{C}^{\prime \prime} .
\end{aligned}
$$

Step 4. Define $\epsilon(v)=1 / \chi(v)$, where $\chi(v)^{\chi(v)}=v=v(\mathcal{A}, \mathcal{B})$. If $\min \left\{\epsilon_{1}^{\mathcal{A}}, \epsilon_{1}^{\mathcal{B}}\right\}>$ $\epsilon(v)$, we use the decomposition rule given above, which amounts to solving recursively two subproblems (12), (13) of respective volumes:

$$
\begin{aligned}
& v\left(\mathcal{A}, \mathcal{B}^{\prime}\right)=|\mathcal{A}|\left|\mathcal{B}^{\prime}\right|=|\mathcal{A}|\left(1-\epsilon_{1}^{\mathcal{B}}\right)|\mathcal{B}|=\left(1-\epsilon_{1}^{\mathcal{B}}\right) v(\mathcal{A}, \mathcal{B}) \leq(1-\epsilon(v)) v \\
& v\left(\mathcal{A}^{\prime \prime}, \mathcal{B}\right)=\left|\mathcal{A}^{\prime \prime}\right||\mathcal{B}|=\left(1-\epsilon_{1}^{\mathcal{A}}\right)|\mathcal{A}||\mathcal{B}|=\left(1-\epsilon_{1}^{\mathcal{A}}\right) v(\mathcal{A}, \mathcal{B}) \leq(1-\epsilon(v)) v
\end{aligned}
$$


This gives rise to the recurrence

$$
R(v) \leq 1+R\left(\left(1-\epsilon_{1}^{\mathcal{B}}\right) v\right)+R\left(\left(1-\epsilon_{1}^{\mathcal{A}}\right) v\right) \leq 1+2 R((1-\epsilon(v)) v) .
$$

Step 5. Let us now suppose that $\epsilon_{1}^{\mathcal{B}} \leq \epsilon(v)$. In this case, we begin by solving subproblem (12). If $\mathcal{A}, \mathcal{B}^{\prime}$ are not dual in $\mathcal{C}^{\prime}$, we get a point $x$ maximal in $\mathcal{C}^{\prime} \backslash$ $\left[\mathcal{A}^{+} \cup\left(\mathcal{B}^{\prime}\right)^{-}\right]$, and we are done. Otherwise we claim that

$$
\mathcal{A}^{\prime \prime}, \mathcal{B} \text { are dual in } \mathcal{C}^{\prime \prime} \Longleftrightarrow \forall a \in \widetilde{\mathcal{A}}: \mathcal{A}^{\prime \prime}, \mathcal{B}^{\prime \prime} \text { are dual in } \mathcal{C}^{\prime \prime}(a),
$$

where $\widetilde{\mathcal{A}}=\left\{a \in \mathcal{A} \mid a_{1} \leq a_{1}^{o}\right\}$, and $\mathcal{C}^{\prime \prime}(a)=\mathcal{C}_{1}^{\prime \prime} \times\left[a_{2}: u_{2}\right] \times \ldots \times\left[a_{n}: u_{n}\right]$.

Proof of (14). The forward direction does not use (12). Suppose that there is an $x \in \mathcal{C}^{\prime \prime}(a) \backslash\left[\left(\mathcal{A}^{\prime \prime}\right)^{+} \cup\left(\mathcal{B}^{\prime \prime}\right)^{-}\right]$for some $a \in \widetilde{\mathcal{A}}$, then $x_{i} \geq a_{i}$, for $i=2, \ldots, n$. If $x \in\left(\mathcal{B}^{\prime}\right)^{-}$, i.e., $x \leq b$ for some $b \in \mathcal{B}^{\prime}$, then by the definition of $\mathcal{B}^{\prime}, b_{1} \geq a_{1}^{o}$. On the other hand, $a \in \widetilde{\mathcal{A}}$ implies that $a_{1} \leq a_{1}^{o}$. But then,

$$
\left(a_{1}, a_{2}, \ldots, a_{n}\right) \leq\left(a_{1}^{o}, x_{2}, \ldots, x_{n}\right) \leq\left(b_{1}, b_{2}, \ldots, b_{n}\right),
$$

which contradicts the assumed duality condition (9). This shows that $x \in \mathcal{C}^{\prime \prime} \backslash$ $\left[\left(\mathcal{A}^{\prime \prime}\right)^{+} \cup\left(\mathcal{B}^{\prime} \cup \mathcal{B}^{\prime \prime}\right)^{-}\right]$.

For the other direction, let $x \in \mathcal{C}^{\prime \prime} \backslash\left[\left(\mathcal{A}^{\prime \prime}\right)^{+} \cup \mathcal{B}^{-}\right]$. Since $x \notin\left(\mathcal{B}^{\prime}\right)^{-}$and $x=\left(x_{1}, x_{2}, \ldots, x_{n}\right)<y \stackrel{\text { def }}{=}\left(a_{1}^{o}, x_{2}, \ldots, x_{n}\right)$, the vector $y$ is also not covered by $\mathcal{B}^{\prime}$. Thus $y \in \mathcal{C}^{\prime} \backslash\left(\mathcal{B}^{\prime}\right)^{-}$. We conclude therefore, assuming (12), that $y \in \mathcal{A}^{+}$, i.e., there is an $a \in \mathcal{A}$ such that $a \leq y$. But this implies that $a \in \widetilde{\mathcal{A}}$ and hence that $x \in \mathcal{C}^{\prime \prime}(a) \backslash\left[\left(\mathcal{A}^{\prime \prime}\right)^{+} \cup\left(\mathcal{B}^{\prime \prime}\right)^{-}\right]$for some $a \in \widetilde{\mathcal{A}}$.

It follows by (14) that, once we discover that (12) holds, we can reduce the solution of subproblem (13) to solving $|\widetilde{\mathcal{A}}|$ subproblems, each of which has a volume of $v\left(\left|\mathcal{A}^{\prime \prime}\right|,\left|\mathcal{B}^{\prime \prime}\right|\right) \leq \epsilon_{1}^{\mathcal{B}} v(\mathcal{A}, \mathcal{B})$. Thus we obtain the recurrence

$$
R(v) \leq 1+R\left(\left(1-\epsilon_{1}^{\mathcal{B}}\right) v\right)+|\mathcal{A}| R\left(\epsilon_{1}^{\mathcal{B}} v\right) \leq R\left(\left(1-\epsilon_{1}^{\mathcal{B}}\right) v\right)+\frac{v}{2} R\left(\epsilon_{1}^{\mathcal{B}} v\right),
$$

where the last inequality follows from $|\mathcal{A}| \leq v / 3$ and $v \geq 9$.

Step 6. Finally, if $\epsilon_{1}^{\mathcal{A}} \leq \epsilon(v)<\epsilon_{1}^{\mathcal{B}}$, we solve subproblem (13), and if we discover that $\mathcal{A}^{\prime \prime}, \mathcal{B}$ are dual in $\mathcal{C}^{\prime \prime}$, we obtain the following rule, symmetric to (14):

$$
\mathcal{A}, \mathcal{B}^{\prime} \text { are dual in } \mathcal{C}^{\prime} \Longleftrightarrow \forall b \in \widetilde{\mathcal{B}}: \mathcal{A}^{\prime}, \mathcal{B}^{\prime} \text { are dual in } \mathcal{C}^{\prime}(b) \text {, }
$$

where $\widetilde{\mathcal{B}}=\left\{b \in \mathcal{B} \mid b_{1} \geq a_{1}^{o}-1\right\}$, and $\mathcal{C}^{\prime}(b)=\mathcal{C}_{1}^{\prime} \times\left[l_{2}: b_{2}\right] \times \ldots \times\left[l_{n}: b_{n}\right]$. This reduces our original problem into one subproblem of volume $\leq\left(1-\epsilon_{1}^{\mathcal{A}}\right) v$, plus $|\widetilde{\mathcal{B}}|$ subproblems, each of volume at most $\epsilon_{1}^{\mathcal{A}} v$, thus giving the recurrence

$$
R(v) \leq 1+R\left(\left(1-\epsilon_{1}^{\mathcal{A}}\right) v\right)+|\mathcal{B}| R\left(\epsilon_{1}^{\mathcal{A}} v\right) \leq R\left(\left(1-\epsilon_{1}^{\mathcal{A}}\right) v\right)+\frac{v}{2} R\left(\epsilon_{1}^{\mathcal{A}} v\right) .
$$

Using induction on $v \geq 9$, it can be shown that the above recurrences imply that $R(v) \leq v^{\chi(v)}$ (see [8]). As $\chi\left(m^{2}\right)<2 \chi(m)$ and $v(\mathcal{A}, \mathcal{B})<m^{2}$, we get $\chi(v)<\chi\left(m^{2}\right)<2 \chi(m) \sim 2 \log m / \log \log m$. Let us also note that every step above can be implemented in at most $O\left(n^{3} m\right)$ time, independent of the chains sizes $\left|\mathcal{C}_{i}\right|$. This establishes the bound stated in Theorem 4 . 


\section{References}

1. R. Agrawal, H. Mannila, R. Srikant, H. Toivonen and A. I. Verkamo, Fast discovery of association rules, In U. M. Fayyad, G. Piatetsky-Shapiro, P. Smyth and R. Uthurusamy eds., Advances in Knowledge Discovery and Data Mining, 307-328, AAAI Press, Menlo Park, California, 1996.

2. J. C. Bioch, Dualization, decision lists and identification of monotone discrete functions, Annals of Mathematics and Artificial Intelligence 24 (1998) 69-91.

3. J. C. Bioch and T. Ibaraki, Complexity of identification and dualization of positive Boolean functions, Information and Computation 123 (1995) 50-63.

4. E. Boros, K. Elbassioni, V. Gurvich and L. Khachiyan, An incremental RNC algorithm for generating all maximal independent sets in hypergraphs of bounded dimension. DIMACS Technical Report 2000-21, Rutgers University. To appear in Parallel Processing Letters.

5. E. Boros, V. Gurvich, L. Khachiyan and K.Makino, Generating partial and multiple transversals of a hypergraph, In: Automata, Languanges and Programming, 27th International Colloquium, ICALP 2000 (Montanari, J.D.P. Rolim and E. Welzl, eds.), pp. 588-599 (Springer Verlag, Berlin, Heidelberg, New York, July 2000). An extended version of this paper is to appear in SIAM J. Computing.

6. Y. Crama, P. L. Hammer and T. Ibaraki, Cause-effect relationships and partially defined boolean functions, Annals of Operations Research 16 (1988) 299-326.

7. T. Eiter and G. Gottlob, Identifying the minimal transversals of a hypergraph and related problems, SIAM Journal on Computing, 24 (1995) 1278-1304.

8. M. L. Fredman and L. Khachiyan, On the complexity of dualization of monotone disjunctive normal forms, J. Algorithms, 21 (1996) 618-628.

9. D. Gunopulos, R. Khardon, H. Mannila, and H. Toivonen, Data mining, hypergraph transversals and machine learning. In: Proceedings of the 16th ACMSIGACT-SIGMOD-SIGART Symposium on Principles of Database Systems, (1997) pp. 12-15.

10. D. S. Johnson, M. Yannakakis and C. H. Papadimitriou, On generating all maximal independent sets, Information Processing Letters, 27 (1988) 119-123.

11. E. Lawler, J. K. Lenstra and A. H. G. Rinnooy Kan, Generating all maximal independent sets: NP-hardness and polynomial-time algorithms, SIAM Journal on Computing, 9 (1980) 558-565.

12. K. Makino and T. Ibaraki, Interor and exterior functions of Boolean functions, Discrete Applied Mathematics, 69 (1996) 209-231.

13. Mangasarian, Mathematical programming in machine learning, in G. Di. Pillo and F. Giannessi eds. Nonlinear Optimization and Applications (Plenum Publishing, New York, 1996) 283-295.

14. A. Prékopa, Stochastic Programming, (Kluwer, Dordrecht, 1995). 